\title{
AAVshRNA-Mediated Suppression of PTEN in Adult Rats in Combination with Salmon Fibrin Administration Enables Regenerative Growth of Corticospinal Axons and Enhances Recovery of Voluntary Motor Function after Cervical Spinal Cord Injury
}

\author{
(1) Gail Lewandowski $\mathbf{i}^{1,2}$ and Oswald Steward ${ }^{1,2,3,4}$ \\ ${ }^{1}$ Reeve-Irvine Research Center, ${ }^{2}$ Department of Anatomy and Neurobiology, ${ }^{3}$ Department of Neurobiology and Behavior, and ${ }^{4}$ Department of \\ Neurosurgery, University of California, Irvine, California 92697
}

Conditional genetic deletion of phosphatase and tensin homolog (PTEN) in the sensorimotor cortex of neonatal mice enables regeneration of corticospinal tract (CST) axons after spinal cord injury (SCI). The present study addresses three questions: (1) whether PTEN knockdown in adult rats by nongenetic techniques enables CST regeneration, (2) whether interventions to enable CST regeneration enhance recovery of voluntary motor function, and (3) whether delivery of salmon fibrin into the injury site further enhances CST regeneration and motor recovery. Adult rats were trained in a staircase-reaching task and then received either intracortical injections of AAVshPTEN to delete PTEN or a control vector expressing shRNA for luciferase (AAVshLuc). Rats then received cervical dorsal hemisection injuries and salmon fibrin was injected into the injury site in half the rats, yielding four groups (AAVshPTEN, AAVshLuc, AAVshPTEN + fibrin, and AAVshLuc + fibrin). Forepaw function was assessed for 10 weeks after injury and CST axons were traced by injecting biotin-conjugated dextran amine into the sensorimotor cortex. Rats that received AAVshPTEN alone did not exhibit improved motor function, whereas rats that received AAVshPTEN and salmon fibrin had significantly higher forelimb-reaching scores. Tract tracing revealed that CST axons extended farther caudally in the group that received AAVshPTEN and salmon fibrin versus other groups. There were no significant differences in lesion size between the groups. Together, these data suggest that the combination of PTEN deletion and salmon fibrin injection into the lesion can significantly improve voluntary motor function after SCI by enabling regenerative growth of CST axons.

Key words: biomaterials; combination therapy; PTEN; regeneration; spinal cord injury

\section{Introduction}

Spinal cord injury (SCI) disrupts descending motor pathways, resulting in loss of voluntary motor function. Damaged axons in the spinal cord do not regenerate because of the greatly diminished intrinsic growth capacity of mature neurons and the growth-inhibitory extrinsic lesion environment (Afshari et al., 2009; Ferguson and Son, 2011; Cregg et al., 2014).

\footnotetext{
Received May 16, 2014; revised June 11, 2014; accepted June 12, 2014.

Author contributions: G.L. designed research; G.L. performed research; G.L. and O.S. analyzed data; G.L. and 0.S. wrote the paper.

This work was supported by the National Institutes of Health (Grant R01 NS047718 to 0.S.) and by generous donations from Cure Medical, Research for Cure, and individual donors. We thank Lisa Flanagan for supplying salmon fibrin and technical expertise and Jamie Mizufuka for technical histological assistance.

O.S. is one of the cofounders of a company called "Axonis," which holds options on patents relating to PTEN deletion and axon regeneration. G.L. declares no competing financial interests.

Correspondence should be addressed to either of the following: Dr. Oswald Steward, Reeve-Irvine Research Center, University of California, Irvine, 1105 Gillespie Neuroscience Research Facility, Irvine, CA 92697-4265, E-mail: osteward@uci.edu; or Gail Lewandowski, Ph.D., Reeve-Irvine Research Center, University of California, Irvine, 1216 Gillespie Neuroscience Research Facility, Irvine, CA 92697-4265, E-mail: glewando@uci.edu.

DOI:10.1523/JNEUROSCI.1996-14.2014

Copyright $\odot 2014$ the authors $\quad 0270-6474 / 14 / 349951-12 \$ 15.00 / 0$
}

Numerous strategies are being explored that neutralize inhibitory factors and coax axon growth and synaptic reconnection (McCreedy and Sakiyama-Elbert, 2012; Zhao and Fawcett, 2013). For example, treating the glial scar with Chondroitinase $\mathrm{ABC}$ has been paired with numerous interventions (Zhao and Fawcett, 2013). In addition, biodegradable hydrogels have been combined with other interventions to reduce glial scarring, to provide growth substrates for regenerating axons, for drug and neurotropic factor delivery, and for scaffolding to retain exogenous glial and/or neuronal cells in the injury site for cell replacement therapies (McCreedy and Sakiyama-Elbert, 2012; Krishna et al., 2013).

However, few of the combination therapies to date target neuron-intrinsic diminished regenerative capacity at the level of the neuronal cell body in the brain. Recent studies show that modifying gene expression in brain neurons can enable axon regeneration after SCI in rodents. One such gene is that for phosphatase and tensin analog (PTEN), a negative regulator of Akt/ mTOR that constrains neuronal cell size and axon elongation 
(van Diepen and Eickholt, 2008; Park et al., 2010). Conditional genetic deletion or short hairpin RNA (shRNA) knockdown of PTEN in the cortex of neonatal mice enables axon regeneration after SCI later in life (Liu et al., 2010; Zukor et al., 2013). In terms of potential therapeutic strategies, the key question is whether PTEN suppression in mature neurons can also enable axon regeneration.

In addition, previous SCI studies have involved mice, which do not develop the large cavities that are seen in rats and other species, including humans (Guth et al., 1999). Therefore, rats are a more suitable animal model for exploring combinatorial strategies to enhance axon growth at the injury site.

Although previous studies documented enhanced corticospinal tract (CST) regeneration with neonatal PTEN deletion, robustly growing axons are still impeded by the inhibitory barrier of the lesion site. Therefore, a combined approach involving PTEN deletion at the cell body and manipulations at the lesion site might be more efficacious than either manipulation alone. Salmon fibrin has been shown to support neurite growth (Ju et al., 2007; Uibo et al., 2009) and a recent study (Sharp et al., 2012) reported that injection of salmon fibrin into a thoracic injury site improved bladder and locomotor function recovery.

Accordingly, our study had three goals: (1) to determine whether PTEN knockdown in adult neurons using a nongenetic technique can enable CST regeneration, (2) to assess whether interventions to enable CST regeneration enhance recovery of voluntary motor function, and (3) to test a combinatorial strategy targeting intrinsic growth potential (PTEN deletion) and extrinsic factors at the injury site (salmon fibrin) in a species (rat) that more closely approximates the histopathology of humans.

\section{Materials and Methods}

Our goal was to develop an adeno-associated virus (AAV)-shRNA-based system to allow deletion of PTEN in the motor cortex of adult rats. For this purpose, we first developed an in vitro assay for screening potential shRNAs. We used an exogenous expression system because PTEN is expressed at low levels in growing cells.

Generation of a rat PTEN expression vector. Full-length rat PTEN cDNA was amplified from rat cerebellum cDNA and cloned into the pDsRed-Monomer-C1 expression vector. The following primers were used: 5 '-TCTCGAGCTCAAGCTTATGACAGCCATCATCAAAG AGA-3' (forward) and 5' -TAGATCCGGTGGATCCTCAGACTTTTGTAATTTGTGGC-3' (reverse) (Integrated DNA Technologies). In each primer, the sequence corresponding to the rat PTEN mRNA sequence is underlined. The remaining sequences are specific for the pDsRedMonomer-C1 expression vector and contain a HindIII site (forward primer) and BamHI site (reverse primer). The Advantage cDNA PCR kit (Clontech) was used for amplification and the reaction mixture contained $100 \mathrm{ng}$ of brain cDNA, primers (400 nм each), $10 \mathrm{~mm} \mathrm{dNTP} \mathrm{mix,}$ $1 \times$ Advantage cDNA PCR mix, and $1 \times$ Advantage cDNA PCR polymerase mix. The following cycling conditions were used: hot start at $94^{\circ} \mathrm{C} \times$ $1 \mathrm{~min}, 35$ cycles of $94^{\circ} \mathrm{C} \times 30 \mathrm{~s}, 68^{\circ} \mathrm{C} \times 3.5 \mathrm{~min}$, a final extension at $68^{\circ} \mathrm{C} \times 3.5 \mathrm{~min}$. The amplification product was concentrated in water using the QIAGEN PCR purification kit. Analysis by electrophoresis on a $1 \%$ agarose gel in $1 \times$ Tris/Borate/EDTA indicated a single product of $\sim 1200 \mathrm{bp}$. This product corresponds to the predicted full-length rat PTEN cDNA (rPTEN) with a HindIII site at the $5^{\prime}$ end and a BamHI site at the $3^{\prime}$ end. The rPTEN was then cloned into the HindIII-BamHI site of the pDsRed-Monomer-C1 vector using the Clontech In-Fusion kit to

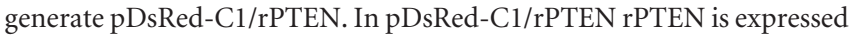
as a fusion to the $\mathrm{C}$ terminus of DsRed-Monomer and expression is driven by the CMV promoter.

Generation of AAV expressing a shRNA that targets PTEN. Four plasmids each with a different predesigned shRNA sequence that targets the rat PTEN gene (shRNA-PTEN), along with two plasmids containing nonspecific shRNA sequences were purchased from SABiosciences (cat- alog \#KR47268G; SureSilencing shRNA plasmids). JM109-competent bacteria (Promega) were transformed with each shRNA plasmid. Large plasmid preparations were made after selecting and validating clones in minipreps. The QIAprep Spin Miniprep kit was used for minipreps and an Endotoxin-free maxi-plasmid prep kit was used for large plasmid preparation (both kits from QIAGEN).

Real-time PCR was used to verify and compare the level of PTEN gene suppression by each candidate shRNA. Briefly, 293T cells were cotransfected with pDsRed-C1/rPTEN and one of the shRNA-PTEN plasmids. Forty-eight hours after transfection, cellular RNA was isolated using TRIzol reagent (Invitrogen). QIAGEN RNase-Free DNase set and the RNeasy MinElute protocol were used for removal of residual DNA and RNA cleanup. The RNA was reverse transcribed into cDNA using the SABiosciences $\mathrm{RT}^{2}$ First Strand Kit (catalog \#C-03). Real-time PCR was performed according to the SABiosciences protocol for using the RT ${ }^{2}$ SYBR Green qPCR Master Mix. The reaction mixture contained $125 \mathrm{ng}$ of CDNA, $400 \mathrm{~nm}$ primer mixture (forward primer, 5' $5^{\prime}$-ACACCGC CAAATTTAACTGC-3' and reverse primer $5^{\prime}$-TACACCAGTCCGTC CTTTCC- $3^{\prime}$ ), and $1 \times$ Master Mix (containing dNTPs). The following cycling conditions were used with an Eppendorf Realplex cycler: denature at $95^{\circ} \mathrm{C} \times 10 \mathrm{~min}, 40 \mathrm{cycles}$ of $95^{\circ} \mathrm{C} \times 15 \mathrm{~s}, 59^{\circ} \mathrm{C} \times 30 \mathrm{~s}, 72^{\circ} \mathrm{C} \times 30 \mathrm{~s}$, and a final extension at $60^{\circ} \mathrm{C} \times 15 \mathrm{~s}$, followed by a melting curve analysis. The percentage suppression of rat PTEN gene expression from pDsRedC1/rPTEN was calculated for each candidate shRNA plasmid. The shRNA-PTEN sequence 3 (5'-GGCACTGTTGTTTCACAAGAT-3') was selected for AAV vector development.

An AAV vector (serotype AAV2/9) expressing shRNA-PTEN sequence 3 from the human U6 promoter and the ZsGreen reporter protein from the CMV promoter (AAVshPTEN) was constructed by the University of Pennsylvania Vector Core. AAV serotype 2/9 was chosen because it has the same neuronal transduction properties as AAV2, but has been shown to have a larger transduction area with more robust transgene expression than AAV2 (Cearley and Wolfe, 2006; Aschauer et al., 2013). In AAVshPTEN, the shRNA that targets PTEN is expressed from the U6 promoter and the ZsGreen reporter gene is expressed from the CMV promoter. In addition, the same AAV2/9 vector construct with the ZsGreen reporter expressing a shRNA that targets luciferase (AAVshLuc) was obtained from the University of Pennsylvania Vector Core for use as a vector control.

Animal use and care. All procedures were approved by the Institutional Animal Care and Use Committee at the University of California-Irvine in compliance with the National Institutes of Health guidelines. Adult female Sprague Dawley rats ( $\sim 180$ g at arrival; Harlan Laboratories) were used in all experiments and maintained on a $12 \mathrm{~h} \mathrm{light/dark} \mathrm{cycle} \mathrm{at} 25^{\circ} \mathrm{C}$. All procedures, including animal testing, were done during the light portion of the cycle.

Studies involving live animals were performed in steps. First, parameters were worked out for AAV-shRNA-mediated PTEN suppression in the cortex of adult rats. Initial studies involved single injections and then multiple injection protocols were tested.

Intracortical injections of $A A V$-shRNA in adult rats. In the first study, rats $(\sim 200 \mathrm{~g})$ received injections of either AAVshPTEN or AAVshLuc using sterile stereotaxic surgical procedures. The rats were anesthetized with an intraperitoneal injection of $80 \mathrm{mg} / \mathrm{kg}$ ketamine and $10 \mathrm{mg} / \mathrm{kg}$ xylazine (Western Medical Supply, Arcadia, CA). The scalp was shaved and swabbed with Betadyne, and a small window of the skull was removed over one hemisphere of the sensorimotor cortex. A total of $10^{9}$ genome copies (GCs) of either AAVshPTEN or AAVshLuc were injected $0.8 \mathrm{~mm}$ below the brain surface at $1 \mathrm{~mm}$ anterior to bregma (AP) and 2 $\mathrm{mm}$ lateral to the midline ( $n=5 \mathrm{rats} / \mathrm{group}$ ). This dose was chosen from a preliminary study as the lowest dose that significantly suppressed PTEN expression and did not cause cytotoxicity. Vectors were injected using a $10 \mu$ l Hamilton microsyringe fitted with a pulled glass micropipette. After completion of the injections, the scalp was sutured and the rats received subcutaneous injections of $10 \mathrm{ml}$ of $0.9 \%$ saline for hydration, $2.5 \mathrm{mg} / \mathrm{kg}$ of the antibiotic Baytril, and $0.01 \mathrm{mg} / \mathrm{kg}$ buprenorphine for pain control. The rats were then placed on soft bedding on a $37^{\circ} \mathrm{C}$ warming blanket until they were fully awake. The rats were monitored daily for 


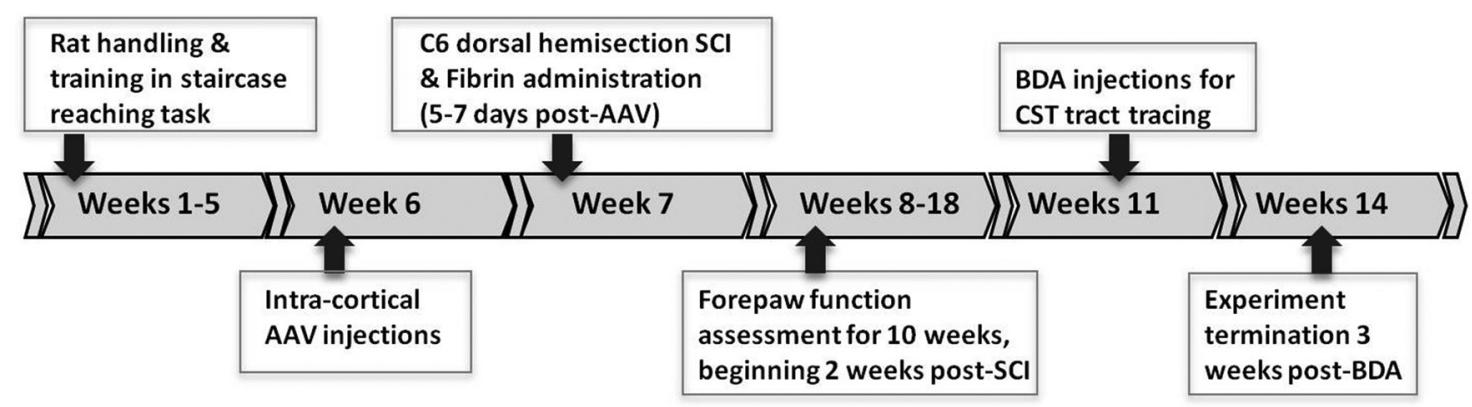

Figure 1. Experiment overview and timeline for the main proof-of-concept experiment. Boxes indicate manipulations during the different time periods on the timeline.

adverse effects of the AAV vector injections. Observations included grooming, eating, drinking, weight, and locomotion.

In the second study, $10^{9} \mathrm{GCs}$ of AAVshPTEN were injected into 5 sites in 1 hemisphere of the sensorimotor cortex ( $n=5$ rats). Injection coordinates were chosen based on previous retrograde tracing of the CST cells of origin from the cervical spinal cord (Strong et al., 2009; Nielson et al., 2011) and studies using cortical stimulation to map the rat forepaw (Neafsey et al., 1986; Fonoff et al., 2009). Each injection was made 0.8 $\mathrm{mm}$ below the brain surface at the following coordinates with respect to bregma: (1) $2 \mathrm{~mm}$ anterior, $2.5 \mathrm{~mm}$ lateral; (2) $1 \mathrm{~mm}$ anterior, $3.2 \mathrm{~mm}$ lateral; (3) $1 \mathrm{~mm}$ anterior, $2.5 \mathrm{~mm}$ lateral; (4) $0 \mathrm{~mm}$ anterior, $3.2 \mathrm{~mm}$ lateral; and (5) $0 \mathrm{~mm}$ anterior, $2.5 \mathrm{~mm}$ lateral. In each injection, the AAVshPTEN was administered in a volume of $1 \mu \mathrm{l}$ over 2-3 min.

Overall plan for the main proof-of-concept experiment. The experimental timeline for the main proof-of-concept experiment is summarized in Figure 1. Adult female rats $(n=60)$ received an identifying number upon arrival (1-60). Rats were trained in the staircase skilled-reaching task for 4 weeks and then received intracortical injections of AAVshPTEN or AAVshLuc. Injections were made into five sites as described in the preceding paragraph on the side contralateral (CL) to the rat's preferred paw (determination of preferred paw is explained below). Injection surgeries were performed over a $3 \mathrm{~d}$ period by 2 different investigators, alternating between AAVshPTEN and AAVshLuc on different days.

Five to $7 \mathrm{~d}$ after injection, rats received a dorsal hemisection injury at cervical level 6 (C6). In each AAVshRNA group, some of the rats also received an injection of salmon fibrin into the lesion site immediately after the injury. Forelimb function was tested from 2 weeks through 10 weeks after SCI; rats then received intracortical injections of biotinconjugated dextran amine (BDA) to trace the CST and were killed humanely $21 \mathrm{~d}$ later.

Forepaw motor function training and assessment. Rats were trained for the staircase skilled-reaching task (catalog \#80300 chambers; Lafayette Instrument Company) using the color pellet variation as described previously (Montoya et al., 1991; Kloth et al., 2006; Klein and Dunnett, 2012). The food pellets ( $45 \mathrm{mg}$, catalog F0021; Bioserve) were colored with a gel-based food paste from AmeriColor (12-pack kit and bright white). Rats (see Animal Attrition section for animal numbers) were food deprived the night before behavioral sessions and food was returned when the behavioral session was completed. Before a behavioral session, both sides of a seven-step staircase were baited with food pellets of various colors (Fig. 2A). During the task, the rat moved from an antechamber onto a narrow platform above the staircase and reached for the food pellets (Fig. 2B). A successful reach is one in which the rat uses their digits to grasp and eat a pellet. During an attempt, the rat may eat the pellet (success), drop the pellet onto a different step (misplaced), or drop the pellet into the chamber such that it can no longer be retrieved (displaced). The difficulty of the task increases as the steps descend.

The following training schedule was used: day 1, 2 sessions of $5 \mathrm{~min}$ with an intersession time of 35-45 min; day 2, 1 session of $10 \mathrm{~min}$; and the remaining training days, 1 session of $15 \mathrm{~min}$ on alternating days for $3 \mathrm{~d}$ a week. After the session ended, the number of pellets eaten, misplaced, and displaced from each step was counted. During the training sessions, the rats were able to use both paws to reach for pellets. In most cases, a rat would use one paw more than the other paw to successfully

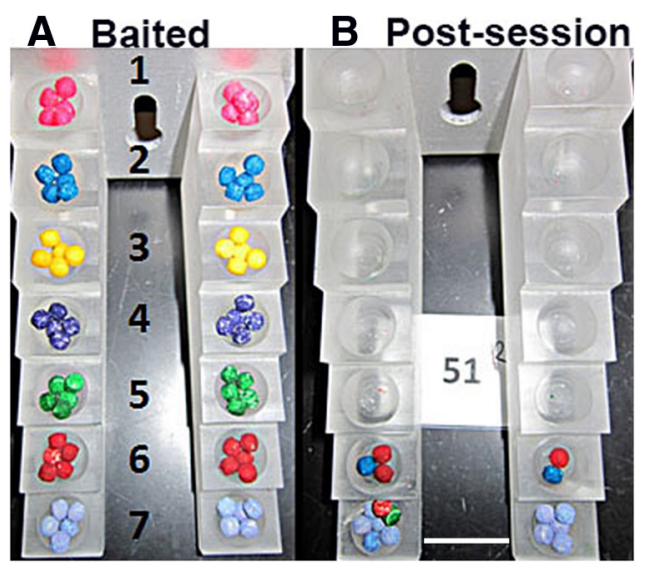

Figure 2. Staircase-reaching task with color pellets. The images illustrate a fully baited staircase viewed from above $(\boldsymbol{A})$ and a view of a staircase after testing showing remaining food pellets $(\boldsymbol{B})$. Lower stairs with two or more colors indicate pellets from higher stairs that were dropped. Scale bar, $2 \mathrm{~mm}$.

reach for the pellets (Table 1). Therefore, the paw that a rat used most successfully was identified as the preferred paw.

Because pretreatment baseline performance was identified as an extraneous variable, the rats were divided into four groups such that the mean behavioral performances of the preferred paw in the last four training sessions were equivalent between the groups. This was done by calculating the mean percentage reaching success (\%Success) at steps 1-6 for each rat and then ranking the rats in descending order of \%Success. Proceeding down the list, each rat was sequentially assigned a number 1-4. The mean scores between groups were analyzed using ANOVA. Group composition was adjusted until the mean scores were equivalent.

After SCI, forepaw motor function was assessed three times a week for 10 weeks and the wells of the staircase were baited with five pellets to increase the difficulty of the task and to ensure statistical separation of groups. Task performance for both training and assessment was expressed as \%Success for each step as follows: (\# pellets eaten/\# pellets baited) $\times 100$.

SCI surgery and salmon fibrin treatment. For animal numbers, see the Animal Attrition section. Rats received AAV injections and, 5-7 d later, received a C6 dorsal hemisection injury. Rats were anesthetized with an intraperitoneal injection of $80 \mathrm{mg} / \mathrm{kg}$ ketamine and $10 \mathrm{mg} / \mathrm{kg}$ xylazine. The hair overlying the cervical to the midthoracic area was removed by shaving and the exposed skin was sterilized with Betadyne. A small midline incision was made through the skin at the C3-T2 vertebral level and underlying connective and muscle tissue were bluntly dissected to expose the cervical portion of the spinal column. A laminectomy was performed at the C6 vertebral level. A dorsal hemisection was created using a Micro Feather Ophthalmic Scalpel with a 15 degree angle blade (catalog \#72045-15; Electron Microscopy Sciences) by extending the cut ventrally to the central canal to ensure ablation of all dorsal pathways.

The following steps were taken to standardize the lesions: (1) a $1 \mathrm{~mm}$ mark was placed on the blade to ensure that the same depth was achieved 
Table 1. Paw preference and $\mathrm{SCl}$ surgery order of rats used in forepaw assessment

\begin{tabular}{|c|c|c|c|}
\hline Rat ID & Paw preference $^{a}$ & Group & SCl surgery order \\
\hline 120206_49 & Right & AAV-shLuc & 1 \\
\hline 120206_39 & Right & AAV-shPTEN & 3 \\
\hline 120206_40 & Right & AAV-shPTEN & 4 \\
\hline 120206_03 & Right & AAV-shLuc & 5 \\
\hline 120206_45 & Left & AAV-shPTEN & 6 \\
\hline 120206_47 & Left & AAV-shLuc & 7 \\
\hline 120206_46 & Left & AAV-shPTEN & 8 \\
\hline 120206_48 & Right & AAV-shLuc & 9 \\
\hline 120206_09 & Right & AAV-shLuc & 10 \\
\hline 120206_57 & Right & AAV-shPTEN & 11 \\
\hline 120206_16 & Right & AAV-shPTEN & 13 \\
\hline 120206_17 & Left & AAV-shLuc & 15 \\
\hline 120206_30 & Right & AAV-shPTEN & 16 \\
\hline 120206_33 & Right & AAV-shLuc & 17 \\
\hline 120206_54 & Left & AAV-shPTEN & 18 \\
\hline 120206_56 & Right & AAV-shLuc & 19 \\
\hline 120206_23 & Left & AAV-shPTEN & 20 \\
\hline 120206_36 & Left & AAV-shLuc & 23 \\
\hline 120206_50 & Left & AAV-shPTEN/Fibrin & 24 \\
\hline 120206_51 & Right & AAV-shPTEN/Fibrin & 25 \\
\hline 120206_37 & Left & AAV-shLuc/Fibrin & 26 \\
\hline 120206_42 & Left & AAV-shPTEN/Fibrin & 27 \\
\hline 120206_43 & Right & AAV-shLuc/Fibrin & 28 \\
\hline 120206_04 & Right & AAV-shLuc/Fibrin & 29 \\
\hline 120206_08 & Right & AAV-shPTEN/Fibrin & 30 \\
\hline 120206_06 & Right & AAV-shPTEN/Fibrin & 31 \\
\hline 120206_20 & Right & AAV-shPTEN/Fibrin & 33 \\
\hline 120206_07 & Left & AAV-shLuc/Fibrin & 34 \\
\hline 120206_58 & Left & AAV-shLuc/Fibrin & 35 \\
\hline 120206_60 & Right & AAV-shLuc/Fibrin & 36 \\
\hline 120206_52 & Left & AAV-shPTEN/Fibrin & 37 \\
\hline 120206_44 & Right & AAV-shPTEN/Fibrin & 38 \\
\hline 120206_25 & Left & AAV-shLuc/Fibrin & 39 \\
\hline 120206_05 & Left & AAV-shPTEN/Fibrin & 40 \\
\hline 120206_27 & Left & AAV-shPTEN/Fibrin & 41 \\
\hline 120206_29 & Right & AAV-shLuc/Fibrin & 42 \\
\hline 120206_12 & Right & AAV-shPTEN/Fibrin & 44 \\
\hline 120206_24 & Left & AAV-shLuc/Fibrin & 47 \\
\hline
\end{tabular}

${ }^{a}$ For each rat, the paw with the highest success rate.

for each lesion, (2) the blade was passed through the lesion three times to ensure that all of the dorsal fibers were ablated, and (3) all injuries were created by one surgeon to reduce variability.

Surgical procedures were completed over $2 \mathrm{~d}$. After completion of the dorsal hemisection injury, rats either received an injection of salmon fibrin into the lesion or no injection. The order of surgery was preplanned to alternate between the AAVshPTEN- and AAVshLuc-injected rats; however, due to surgery issues, including anesthesia and death, the order was not strictly maintained (Table 1 ). In addition, all salmon fibrin injections were done the same day to maintain consistency in the salmon fibrin preparation across animals. The surgeon did not know the vector group assignment, only whether or not the rat was to receive a salmon fibrin injection.

Salmon fibrin was prepared as described previously (Sharp et al., 2012). Briefly, salmon fibrinogen $(3.0 \mathrm{mg} / \mathrm{ml})$ and thrombin $(0.15 \mathrm{U} / \mathrm{ml}$; Sea Run Holdings) were diluted into phenol-free EMEM and gently mixed. Approximately $5-10 \mu \mathrm{l}$ of the liquid mixture was carefully injected into the lesion site using a Hamilton syringe. A preliminary experiment was done to determine the fibrinogen dilution and thrombin concentration that would result in sufficient polymerization (fibrin formation) within 2-3 min. Rats were maintained in a stationary position for at least $5 \mathrm{~min}$ after the fibrin injection into the lesion. Fibrin solution from each injection was also placed on a surface and monitored visually to ensure that complete polymerization had occurred.

Post-operative care. After completing the dorsal hemisections and injections, muscles were sutured in layers with 4-0 chromic gut (catalog
Table 2. Experiment 1: Animal attrition and exclusions

\begin{tabular}{lll}
\hline Experimental step & Attrition & Remaining \\
\hline Starting no. of rats & - & 60 \\
Rats that did not meet performance criterion & 7 & 53 \\
Rats that died during vector administration & 0 & 53 \\
Rats that died during SCl surgery & 4 & 49 \\
Rats that removed for autophagia & 5 & 44 \\
Rats with incomplete SCl & 6 & 38 \\
Total no. of rats for behavioral analysis & & 38 \\
\hline
\end{tabular}

\#G181H; Ethicon) and the skin was closed with wound clips (Fisher Scientific). Postoperatively, rats received subcutaneous injections of 10 $\mathrm{ml}$ of $0.9 \%$ saline for hydration for $10 \mathrm{~d}, 2.5 \mathrm{mg} / \mathrm{kg}$ Baytril to reduce the risk of bladder infection for $7 \mathrm{~d}$, and $0.01 \mathrm{mg} / \mathrm{kg}$ buprenorphine for pain control for $7 \mathrm{~d}$. Bladders were manually expressed twice daily until each rat regained autonomic bladder function ( $\sim 2$ weeks after injury). Rats were placed on water-circulating, jacketed warming pads at $37^{\circ} \mathrm{C}$ to maintain body temperature for 3-7 d post-injury (dpi). Rats were group housed (four per cage) and monitored twice daily for general health, coat quality (indicative of grooming activity), and mobility within the cage. Rats were also monitored for signs of skin lesions on the paralyzed limbs or autophagia of the toes. If a rat exhibited severe autophagia, it was killed (see attrition table for incidence of autophagia).

Anterograde tracing of CST projections. CST projections were traced by injecting BDA (10,000 MW, lysine fixable; Invitrogen) into the sensorimotor cortex. Rats were anesthetized with an intraperitoneal injection of $80 \mathrm{mg} / \mathrm{kg}$ ketamine and $10 \mathrm{mg} / \mathrm{kg}$ xylazine. The hair on the scalp was shaved and swabbed with Betadyne. Using a stereotaxic device and a 10 $\mu l$ Hamilton microsyringe fitted with a pulled glass micropipette, BDA was injected into five sites in the sensorimotor cortex at the same coordinates used for AAV vector administration. The rats were humanely killed $21 \mathrm{~d}$ after BDA with an intraperitoneal injection of Euthasol (195 $\mathrm{mg} / \mathrm{ml}$ pentobarbital sodium and $25 \mathrm{mg} / \mathrm{ml}$ phenytoin sodium; Delmarva Laboratories) and transcardially perfusion with $4 \%$ paraformaldehyde in $0.1 \mathrm{M}$ phosphate, $\mathrm{pH}$ 7.4. The brains and spinal cords were removed, taking care not to disrupt scar tissue at the lesion site, and postfixed in the same fixative for $4 \mathrm{~h}$ at $4^{\circ} \mathrm{C}$.

Animal attrition, randomization, and blinding. A number of rats died or were removed from the study at various stages (Table 2). After the completion of behavioral training, AAV vector administration, SCI surgery, and lesion completeness evaluation, the numbers of rats for motor function recovery analysis were as follows: (1) AAVshPTEN/Fibrin $(n=$ 11), (2) AAVshPTEN $(n=9)$, (3) AAVshLuc/Fibrin $(n=9)$, and (4) AAVshLuc $(n=9)$. The researcher doing the post-SCI functional assessment was blind to the treatment group membership of any particular rat. In addition, rats were removed from the post-SCI portion of the study for autophagia without knowledge of group membership.

High incidence of autophagia in a follow-up experiment. We performed a second experiment that was in part an internal replication and also was designed to assess whether co-deletion of PTEN and SOCS3 was more effective than deletion of PTEN alone. This experiment did not yield interpretable data because of a very high incidence of autophagia, which we speculate was due to a change in the way the lesions were performed. We report the experiment here to alert other investigators about the possible occurrence of autophagia in this experimental paradigm.

Forty-eight adult female rats of $\sim 250 \mathrm{~g}$ were trained for 4 weeks in the staircase-reaching task and then divided into 3 groups of 16 rats based on performance, as described for Experiment 1. Groups were as follows: (1) AAVshPTEN + salmon fibrin, (2) AAVshPTEN + AAVshSOCS3 + salmon fibrin, and (3) lesion-only controls.

Intracortical injections of AAVshRNA vectors or saline $(1.5 \mu \mathrm{l})$ were injected $0.8 \mathrm{~mm}$ below the brain surface over $6 \mathrm{~min}$ using a syringe pump at the following coordinates relative to bregma: (1) $1 \mathrm{~mm}$ anterior, 2.5 mm lateral; (2) $2.5 \mathrm{~mm}$ anterior, $2.5 \mathrm{~mm}$ lateral; (3) $1 \mathrm{~mm}$ anterior, 3.2 $\mathrm{mm}$ lateral; and (4) $2.5 \mathrm{~mm}$ anterior, $3.2 \mathrm{~mm}$. Immediately after vector administration, the rats received a C6 dorsal hemisection using a different procedure than that described in SCI surgery and salmon fibrin treat- 
Table 3. Experiment 2: Animal attrition and incidence and timing of autophagia

\begin{tabular}{|c|c|c|c|c|c|}
\hline Rat & Group & Day of onset & Site & Relative to vector $^{a}$ & Severity \\
\hline 27 & shPTEN-Fibrin & 4 & Forepaw & Contralateral & Mild \\
\hline 31 & Untreated & 4 & Forepaw & Contralateral & Severe \\
\hline 33 & shPTEN-Fibrin & 7 & Forepaws & Both & Moderate \\
\hline 18 & shPTEN-Fibrin & 11 & Forepaw & Contralateral & Severe \\
\hline 8 & shPTEN/shSOCS3-Fibrin & 13 & Forepaw & Contralateral & Mild \\
\hline 37 & shPTEN/shSOCS3-Fibrin & 13 & Forepaw & Contralateral & Moderate \\
\hline 8 & shPTEN/shSOCS3-Fibrin & 14 & Forepaw & Ipsilateral & Mild \\
\hline 14 & shPTEN-Fibrin & 14 & Forepaw & Ipsilateral & Mild \\
\hline 19 & shPTEN-Fibrin & 14 & Forepaw & Contralateral & Mild \\
\hline 5 & Untreated & 15 & Forepaw & Contralateral & Mild \\
\hline 2 & shPTEN/shSOCS3-Fibrin & 19 & Hindpaw & Contralateral & Mild \\
\hline 25 & Untreated & 22 & Forepaw & Ipsilateral & Mild \\
\hline 9 & shPTEN/shSOCS3-Fibrin & 24 & Forepaw & Contralateral & Mild \\
\hline 24 & shPTEN-Fibrin & 24 & Hindpaw & Contralateral & Moderate \\
\hline 26 & shPTEN/shSOCS3-Fibrin & 24 & Forepaw & Contralateral & Moderate \\
\hline 40 & Untreated & 28 & Hindpaw & Contralateral & Severe \\
\hline 10 & Untreated & 29 & Hindpaw & Ipsilateral & Mild \\
\hline 41 & shPTEN/shSOCS3-Fibrin & 30 & Forepaw & Contralateral & Mild \\
\hline 46 & shPTEN/shSOCS3-Fibrin & 30 & Hindpaw & Ipsilateral & Mild \\
\hline 18 & shPTEN-Fibrin & 31 & Hindpaw & Ipsilateral & Moderate \\
\hline 21 & Untreated & 34 & Forepaw & Ipsilateral & Mild \\
\hline 12 & shPTEN-Fibrin & 35 & Hindpaw & Contralateral & Mild \\
\hline 30 & Untreated & 39 & Forepaw & Contralateral & Moderate \\
\hline 4 & shPTEN-Fibrin & 46 & Hindpaw & Contralateral & Mild \\
\hline 34 & shPTEN-Fibrin & 48 & Forepaw & Contralateral & Mild \\
\hline 44 & shPTEN/shSOCS3-Fibrin & 54 & Forepaw & Ipsilateral & Mild \\
\hline 31 & Untreated & 62 & Hindpaw & Contralateral & Moderate \\
\hline 30 & Untreated & 65 & Forepaw & Ipsilateral & Moderate \\
\hline 48 & Untreated & 66 & Hindpaw & Ipsilateral & Moderate \\
\hline 41 & shPTEN/shSOCS3-Fibrin & 72 & Hindpaw & Ipsilateral & Mild \\
\hline 42 & shPTEN-Fibrin & 72 & Hindpaw & Ipsilateral & Mild \\
\hline 45 & shPTEN/shSOCS3-Fibrin & 72 & Hindpaw & Ipsilateral & Mild \\
\hline 46 & shPTEN/shSOCS3-Fibrin & 83 & Forepaw & Ipsilateral & Mild \\
\hline
\end{tabular}

${ }^{a}$ Laterality in terms of AAV injection.

ment, above, in that rats were secured in a stereotaxic device using the ear bars and a tail clamp to stabilize the rat and spinal cord and hemisections were done with a \#11 blade attached to the stereotaxic rather than by hand. To create the lesions, the dorsal surface of the spinal cord was lightly scored with a feather blade to provide a guide for the dorsal hemisection and the \#11 blade was lowered $1.9 \mathrm{~mm}$ (relative to the spinal cord dorsal surface) with the sharp side near the lateral edge of the spinal cord. The blade was then moved horizontally in the medial-lateral plane across the spinal cord to generate a dorsal hemisection lesion. This step was repeated three times to ensure a complete lesion. Rats in Groups 1 and 2 received salmon fibrin injections into the lesion after injury, as in Experiment 1.

Attrition. Of the 48 rats in Experiment 2, nine died during surgery or within a week after injury and three had incomplete injuries and were removed from the study. Of the 36 rats remaining, there were 13 in the AAVshPTEN/fibrin group, 13 in the AAVshPTEN-AAVSOCS3/fibrin group, and 10 rats in the untreated group. During the post-injury assessment period, there was a very high incidence of mild to severe autophagia of forelimbs and hindlimbs. All incidences of autophagia required an Elizabethan collar to prevent further autophagia. Severity of autophagia was defined as follows: mild, paw chewing with no digit loss, Elizabethan collar could be removed for $30 \mathrm{~min}$ without new autophagia; moderate, complete or partial loss of 1-2 digits, required Elizabethan collar could be removed for $30 \mathrm{~min}$ without new autophagia; or severe, complete or partial loss of $>2$ digits and constant need of Elizabethan collar. Autophagia began within the first week after injury and the number of new autophagia incidences (hindlimb and/or forelimb) increased steadily until 5 weeks after injury (Table 3). After 5 weeks, there were 1-3 new autophagia incidences per week. By the end of the study, 26 of 36 rats had exhibited at least mild autophagia of their limbs and had been excluded from the functional analysis. The distribution of autophagia across the three groups was similar (Group 1, 8 of 13 rats; Group 2, 10 of 13 rats; and Group 3, 8 of 10 rats). Because the high incidence of autophagia resulted in low animals per group numbers, meaningful statistical analysis of functional recovery was not possible.

Histology. Brains and spinal cords were cryoprotected in $27 \%$ sucrose, rapidly frozen in TissueTek optimal cutting temperature medium (VWR International), and stored at $-20^{\circ} \mathrm{C}$. Tissue was sectioned on a cryostat at $20 \mu \mathrm{m}$ and sections were collected and stored in PBS with $0.02 \%$ sodium azide. Brains were sectioned in the coronal plane and sections were collected from $\sim-2 \mathrm{~mm}$ AP to just anterior to the olfactory bulb. Spinal cords were divided into three blocks: (1) the injury block of $\sim 15 \mathrm{~mm}$ centered around and spanning the injury site, (2) a rostral block adjacent to the injury block, and (3) a caudal block adjacent to the injury segment. The injury block was sectioned in the sagittal plane. Every section was collected and maintained in serial order in 96-well plates and stored at $4^{\circ} \mathrm{C}$. Rostral and caudal spinal cord segments were sectioned in the coronal plane for assessment of BDA labeling.

Immunohistochemical assessment of PTEN suppression and activation of phosphorylation of ribosomal protein S6. To determine the area of the cortex in which PTEN was suppressed, free-floating brain sections were immunostained for PTEN. All steps, including antibody incubations, were done at room temperature. Sections were washed sequentially in TBS (100 mm Tris, pH 7.4, $150 \mathrm{~mm} \mathrm{NaCl}$ ) and TBST (TBS with $0.05 \%$ Tween 20) $2 \times$ for $10 \mathrm{~min}$. Endogenous peroxidase was quenched by incubation in $3 \% \mathrm{H}_{2} \mathrm{O}_{2} /$ TBS for $15 \mathrm{~min}$. Sections were then washed in TBS for 5 min, blocked in tyramide signal amplification (TSA) blocking reagent (PerkinElmer), and then incubated overnight with rabbit antiPTEN (catalog \#9188S; Cell Signaling Technology) diluted 1:250 (for chromogenic detection) or 1:1000 (for fluorescence detection) in SignalStain diluent (Cell Signaling Technology ). For chromogenic detection, sections were incubated sequentially with the following: (1) biotinylated donkey anti-rabbit IgG (1:500, catalog \#711-065-152; Jackson ImmunoResearch) in TSA blocking buffer, (2) ABC reagent (Vectastain Elite kit, catalog \#PK-6100; Vector Laboratories), (3) DAB (Vector DAB kit, catalog \#SK-4100; Vector Laboratories), (4) counterstained with hematoxylin/eosin (H\&E), (5) dehydrated through graded ethanol and xylene, and (6) coverslipped using DPX mounting medium (Sigma Aldrich). For fluorescence detection, sections were incubated with peroxidaseconjugated donkey anti-rabbit IgG (1:500, catalog \#711-036-152; Jackson ImmunoResearch), TSA-Cy3 diluted 1:100 in amplification diluent (PerkinElmer) for $30 \mathrm{~min}$, counterstained with DAPI (Invitrogen) diluted 1:7500 in TBS for $10 \mathrm{~min}$, and coverslipped using VectaShield mounting medium (Vector Laboratories).

Suppression of PTEN activates AKT signaling, and one downstream indicator is phosphorylation of ribosomal protein S6. To assess S6 phosphorylation, sections were immunostained using an antibody specific for the phosphorylated form of S6 (pS6). Steps up to the primary antibody incubation were the same as for chromogenic detection of PTEN. Sections were then incubated with rabbit anti-pS6 (clone D57.2.2E, catalog \#4858; Cell Signaling Technology) diluted 1:250 in SignalStain diluent overnight. All subsequent steps were as for chromogenic detection of PTEN.

Immunohistochemical staining of neurons with NeuN. Free-floating brain sections were immunostained for NeuN. All steps were done at room temperature. Sections were incubated with PBS, PBS plus Triton X-100 with 5\% normal goat serum (PBST-NGS), and then with PBSTNGS plus mouse anti-NeuN (1:250, A60; Millipore) overnight. After washing in PBST and PBS, sections were incubated with Alexa Fluor 594-conjugated goat anti-mouse IgG (1:500; Invitrogen), washed as described in the preceding paragraphs and coverslipped using VectaShield mounting medium.

Detection of BDA-labeled axons. Floating cross-sections from blocks rostral and caudal to the injury block were stained for BDA (for all 38 rats). We first evaluated the rostral and caudal spinal cord segments for BDA-labeled axons. In six cases, both the rostral and caudal segments were positive for BDA-labeled axons (indicative of an incomplete injury) and were excluded from the functional and histological analyses. Of the six rats with incomplete lesions, three were from the AAVshLuc/fibrin group, two were from the AAVshLuc group, and one was in the AAVshPTEN group. In 14 cases, the rostral segments were either negative 
or had very few BDA-labeled axons and were also excluded from analysis. After these exclusions, the number of cases prepared for assessment of CST regenerative growth were as follows: AAVshPTEN/fibrin, $n=5$; AAVshPTEN, $n=5$; AAVshLuc/fibrin, $n=4$; and AAVshLuc, $n=4$.

The block containing the injury site was sectioned in the sagittal plan and all sections were mounted in serial order onto Superfrost Plus glass slides (Fisher Scientific) and allowed to dry overnight at room temperature. Sections were slide mounted before BDA detection to preserve the architecture of the injury site, which can be lost when floating sections are stained.

For the 18 cases in which the number of BDA-labeled axons was sufficient (as judged from the rostral cross-sections), sagittal sections were stained for BDA. Slides were washed $3 \times$ for 10 min in PBS and endogenous peroxidase activity was quenched by incubation in $1 \% \mathrm{H}_{2} \mathrm{O}_{2}$ in PBS for $15 \mathrm{~min}$. Slides were washed $2 \times$ in PBS, and then incubated in ABC reagent (Elite Vectastain kit) according to the manufacturer's instructions, and prepared in PBST (PBS plus $0.5 \%$ Triton) for $2 \mathrm{~h}$ at room temperature. Slides were washed $2 \times$ in PBST and $2 \times$ in PBS and then incubated with Cy3-TSA diluted 1:100 in amplification solution (PerkinElmer) for $20 \mathrm{~min}$ at room temperature. The slides were washed and coverslipped using the Vectashield mounting medium.

Counting of BDA-labeled axons. Sagittal sections were stained for BDA and imaged using an Olympus VS 110 slide scanner. The images were converted to TIFF files using the OlyVia 2.4 software and then to 8-bit, RGB TIFF files in Adobe Photoshop CS6. Using Photoshop, BDAlabeled axons $\left(\mathrm{BDA}^{+}\right)$were counted at $0.5 \mathrm{~mm}$ intervals relative to the rostral lesion edge. To do this, a grid with vertical lines $0.5 \mathrm{~mm}$ apart was placed over a spinal cord image such that one of the lines was as close as possible to the rostral lesion edge. This line was defined as a distance of 0 $\mathrm{mm}$. Therefore, $\mathrm{BDA}^{+}$axons rostral to the lesion edge were at distances $<0 \mathrm{~mm}$ and $\mathrm{BDA}^{+}$axons caudal to the lesion edge were at distances $>0$ $\mathrm{mm}$. The number of dorsal and ventral $\mathrm{BDA}^{+}$axons that crossed each vertical line in every section was counted. The number of $\mathrm{BDA}^{+}$axons at each interval $x$ were summed across all sections and normalized to the total number of $\mathrm{BDA}^{+}$axons in the rostral spinal cord segment as follows: $\left(\sum \mathrm{BDA}^{+}\right.$axons at interval $\left.x\right) /$ total number of $\mathrm{BDA}^{+}$axons.

Calculation of lesion volumes. Lesion volume assessments were done for the cases in which sagittal sections were stained for BDA (AAVshPTEN/fibrin, $n=7$; AAVshPTEN, $n=5$; AAVshLuc/fibrin, $n=$ 6; and AAVshLuc, $n=5$ ). Serial sections of known separation distances through the injury site were stained with DAPI and the lesion area, including both the cavity and surrounding damaged tissue, was outlined using Photoshop CS6 in an average of 40 sections per spinal cord. The lesion volume for each section was calculated by multiplying the measured area by section thickness. Volumes were then summed for a final estimation of lesion volume in cubic millimeters.

Statistical analysis. Behavioral and histological data were analyzed using ProStat Version 6 software (Poly Software International). Repeated measures two-way ANOVA was used for group comparisons of forepaw performance over time. One-way ANOVA with Bonferroni's correction was used for multiple group comparisons for each time point and each step. Histological data distribution was generally normal and was analyzed using one-way ANOVA with Bonferroni's correction for multiple pairwise comparisons.

\section{Results}

Generation of an AAV vector expressing shRNA targeting rat and human PTEN

Because we could not identify a cell line that expressed suitable levels of rat PTEN, we generated a vector to express rat PTEN
(pDSRed-C1/rPTEN) and transfected 293T cells with this vector. We then assessed the capacity of four candidate shRNA sequences and two nonspecific control sequences to suppress rat PTEN gene expression in pDSRed-C1/rPTEN-expressing cells.

All 4 shRNA sequences suppressed PTEN expression from pDSRed-C1/rPTEN with an average suppression of $86.5 \%$ (SD = $1.3 \%$ ); sequence 3 had the highest level of suppression $(88 \%)$. PTEN was not suppressed by the two nonspecific control sequences $(0.002 \%$ and $0.005 \%$, respectively). Although all shRNA sequences were $100 \%$ homologous to rat PTEN, sequences 3 and 4 were also $100 \%$ homologous to the human PTEN gene. Therefore, shRNA sequence 3 (GGCACTGTTGTTTCACAAGAT) was selected for AAVshPTEN vector production. This shRNA-PTEN sequence is homologous to rat PTEN sequence 573-593 and to human PTEN sequence 1604-1624. In addition, sequence 3 is 95\% homologous to the mouse PTEN gene (1441-1461), with a single mismatch at nucleotide 1448 .

\section{Intracortical injection of AAVshPTEN suppresses PTEN expression in adult rats}

A single cortical injection of AAVshPTEN was made to test the capacity of AAVshPTEN to suppress PTEN in adult rat brain. Expression of the ZsGreen reporter gene was visible at 3 weeks after injection and was used to define the transduction area of both AAVshPTEN and AAVshLuc (Fig. $3 A, D$ ). At high magnification, ZsGreen-positive neurons were observed, indicating transduction of neurons by AAVshPTEN (Fig. 3 A, inset). Using immunohistochemistry, we found suppression of PTEN protein expression at 7 and $10 \mathrm{~d}$ (data not shown), with peak suppression at 3 weeks (Fig. 3B). At 3 weeks after injection, PTEN expression was largely suppressed in the area corresponding to ZsGreen expression (Fig. $3 A, B$ ). Inspection of cortical layer $\mathrm{V}$ in this area at a high magnification revealed that PTEN expression was undetectable in many neurons (Fig. $3 H$ ).

Sampling from 5 animals that received 1 injection revealed that a single intracortical injection of $10^{9}$ GCs of AAVshPTEN suppressed PTEN protein expression in an area $\sim 0.8-0.84 \mathrm{~mm}$ in diameter and $1.35 \mathrm{~mm}$ in dorsal-ventral extent.

Next, we assessed PTEN expression after five spaced injections of AAVshPTEN designed to blanket the forelimb region of the sensorimotor cortex. As illustrated in Figure 4, PTEN expression 


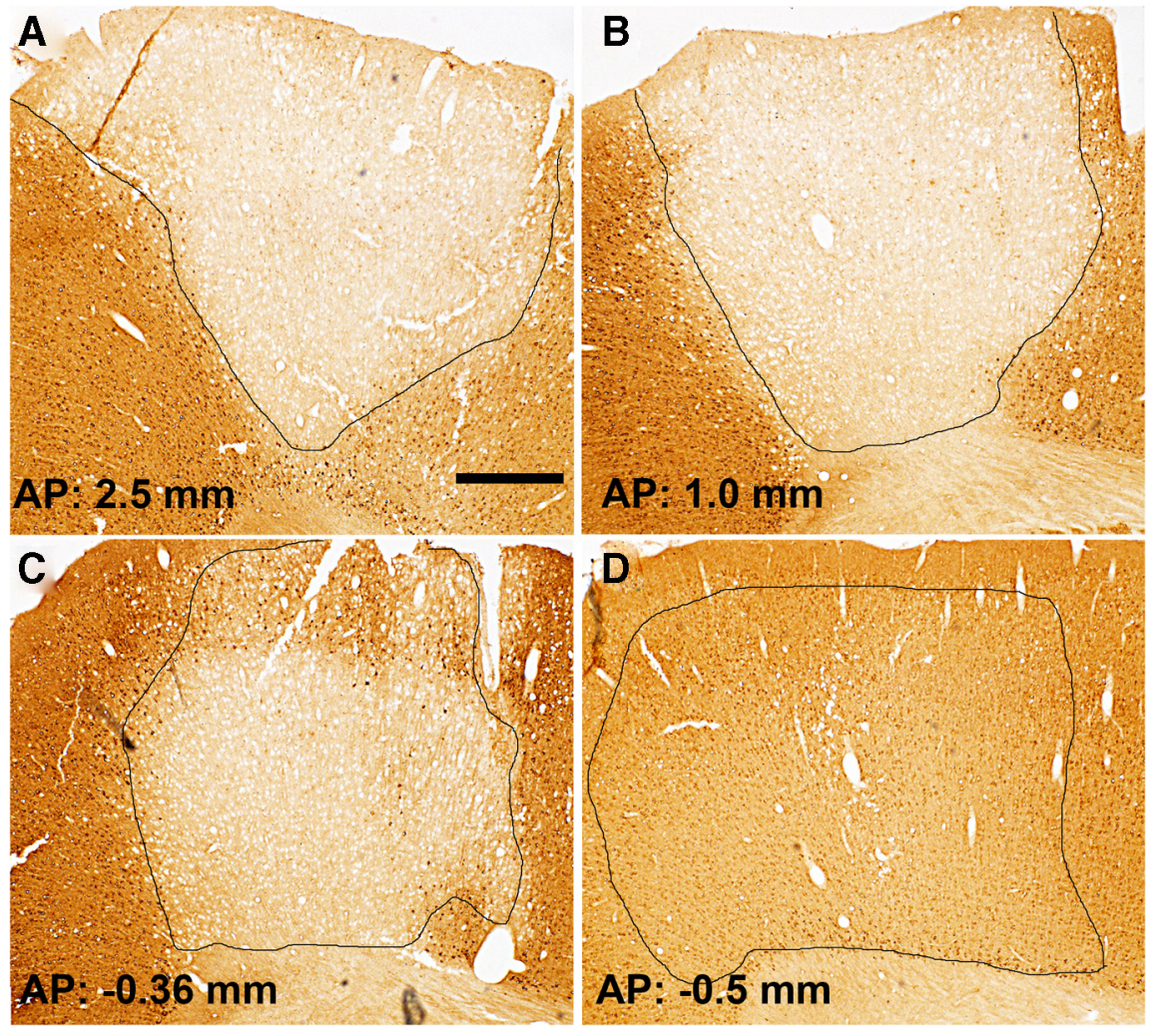

Figure 4. Suppression of PTEN in the sensorimotor cortex of adult rats 3 weeks after 5 injections of AAVshPTEN. A-C, Panels illustrate coronal sections at different rostrocaudal levels through the somatosensory cortex of rats that received AAVshPTEN. The area of PTEN suppression is outlined in black and the approximate coordinates relative to bregma are indicated. D, Area of diminished PTEN suppression, the posterior boundary between the area in which PTEN is suppressed and nearby areas with normal PTEN expression. Scale bars, $0.5 \mathrm{~mm}$.

was suppressed in a large portion of the forelimb region of the sensorimotor cortex at 3 weeks after injection. The area of PTEN suppression extended for $\sim 3 \mathrm{~mm}$ in the anterior-posterior plane and for $\sim 2 \mathrm{~mm}$ in the medial-lateral plane (as measured for cortical layer V). We used the same injection parameters in another set of rats $(n=21)$ for the main proof-of-concept experiment, which lasted 15 weeks after AAVshRNA administration. At the end of the experiment, PTEN gene expression was still greatly suppressed (Fig. 5C-F). NeuN immunostaining of adjacent brain sections (from the same animal) within $200 \mu \mathrm{m}$ of the PTEN-immunostained sections revealed that there was no obvious cell loss in the area of PTEN suppression (Fig. $5 G-I$ ). It is noteworthy that, at this long postinjection interval, some of the cells with ZsGreen expression had the morphology of astrocytes, although a few neurons (positive for NeuN) also expressed ZsGreen (arrows).

PTEN deletion activates the AKT signaling pathway, and a useful downstream indicator of this activation is increased phosphorylation of ribosomal protein S6 (pS6). Consistent with this expectation, pS6 immunostaining was robustly increased in cortical motor neurons in the area of PTEN protein suppression (Fig. $3 C, H$ ). In contrast, rats that received control injections of AAVshLuc showed no suppression of PTEN and no increase in pS6 immunostaining in the AAVshLuc injection area (Fig. 3D-F).

\section{Pre-injury performance in the staircase task}

By the end of the 4 week pre-injury training period, rats reliably performed in the staircase task and were able to retrieve $70 \%$ or more of the pellets from steps 1-4 (Fig. 6). Success dropped off for step 5, and the success rate for step 6 averaged $<10 \%$.

Most rats were more successful with one paw, so data are presented separately for the preferred paw (Fig. 6A) and the nonpreferred paw (Fig. 6B). As noted in the Materials and Methods, groups were compiled by matching preferred paw performance and, as a result, there was more variability between groups for the nonpreferred paw (Fig. 6B). Vector injections were made into the cortex CL to the preferred paw and the paws are hereafter referred to as CL and ipsilateral (IL) to the vector injection.

\section{Rats receiving AAV-shPTEN and salmon fibrin exhibited enhanced recovery of forepaw function after cervical SCI Group performance versus time after injury}

After SCI, reaching performance was assessed separately for the paw CL (CL) to and IL to AAV vector injections for 10 weeks. The percentage success at each step was calculated for each rat. To assess overall group performance at each dpi, the percentage success scores from steps 1-6 for all the rats in a group were combined and averaged and reported as the mean percentage success.

Data were first analyzed as overall group performance for each time after injury (Figs. $7 A, B$ ). The $\mathrm{C} 6$ dorsal hemisection is a bilateral injury of moderate intensity so, as expected, all groups exhibited bilateral deficits in forelimb function, but even at the earliest assessment time after SCI $(14 \mathrm{~d})$, rats in all groups were able to obtain some pellets ( $30 \%$ vs $60 \%$ pre-SCI for the CL paw).

\section{CL paw}

For the CL paw, the performance of all groups was similar until $\sim 22$ dpi. The rats in the AAVshPTEN/fibrin group showed improved CL paw recovery from 22 through $70 \mathrm{dpi}$, with success rates higher than that of the other groups (Fig. $7 A$ ). Analysis of the post-SCI values for the CL forepaw by repeated-measures ANOVA revealed significant differences between groups $(\mathrm{df}=3 ; F=104.8 ; p<0.0001)$ and significant differences over time $(\mathrm{df}=19 ; F=5.72 ; p<0.0001)$. Post hoc comparisons of the groups at each dpi by one-way ANOVA with Bonferroni's correction revealed significant differences between the AAVshPTEN/fibrin group and the other groups on days 22-70 (Fig. 7A, asterisk; see legend for time point statistics). The performances of the other three groups (AAVshLuc, AAVshLuc/fibrin, and AAVshPTEN) were similar to each other. Finally, analysis of each group over all postinjury days by repeated measures one-way ANOVA indicated that the performance of the AAVshPTEN/fibrin group changed significantly over time $(\mathrm{df}=19 ; F=3.448 ; p<$ 0.0001 ). The performance of other groups did not change significantly over the post-injury assessment period. 


\section{IL paw}

Interestingly, the success rate for the IL paw was also greater in the AAVshPTEN/ fibrin group (Fig. 7B). Repeated-measures ANOVA revealed significant differences between groups $(\mathrm{df}=3 ; F=245.8 ; p<$ $0.0001)$ and over time $(\mathrm{df}=19 ; F=3.72$, $p=0.006)$. One-way ANOVA with Bonferroni's multiple comparisons at each dpi revealed that the AAVshPTEN/fibrin group was significantly different from AAVshPTEN and AAVshLuc at $38 \mathrm{dpi}$ and at 57-71 dpi, and from only the AAVshLuc group at $50 \mathrm{dpi}$ and $55 \mathrm{dpi}$ (Fig. $7 B$, asterisk; see legend for statistics for each time point). The AAVshPTEN/ fibrin group was not significantly different from the AAVshLuc/fibrin group, and there were no significant differences between the AAVshLuc, AAVshLuc/fibrin and AAVshPTEN groups. Last, repeatedmeasures one-way ANOVA of the AAVshPTEN/fibrin group indicated that the group's success rate changed significantly over time $(F=3.575, p<0.0001)$. The performance of other groups did not change significantly over the post-injury assessment period.

\section{Group performance by step}

To compare the success rates of the groups at different levels of reaching difficulty, post-SCI reaching data were also analyzed for each step (Figs. 7C,D). After SCI, rats in all groups had lower percentage success than preoperatively (cf. Figs. $7 C, D$, Fig. 6). However, in the AAVshPTEN/fibrin group, the percentage success of the CL forepaw was higher than in all other groups (Fig. 7C). Oneway ANOVA with repeated measures was done separately for each step and revealed significant group differences for the CL paw at steps $1-5$ (step 1: $F=66.86, p<$ 0.0001 ; step 2: $F=47.82, p<0.0001$; step $3: F=25.09, p<0.0001$; step $4: F=14.60$, $p<0.0001$; step 5: $F=5.8, p=0.0007$; and step 6: $F=0.34, p=0.80)$. Post hoc group comparisons by one-way ANOVA with Bonferroni's correction indicated that the success rate of the AAVshPTEN/fibrin group was significantly higher than that of all other groups at steps $1-5(p<0.0001$ for all comparisons; Fig. $7 C$, asterisks).

Step performance analysis for the IL paw by one-way ANOVA with repeated measures (for each step) revealed significant group differences at steps $1-5$ (step $1: F=38.03, p<0.0001$; step $2: F=$ 33.0, $p<0.0001$; step 3: $F=27.24, p<0.0001$; step $4: F=13.48$, $p<0.0001$; step 5: $F=5.4, p=0.0012$; and step $6: F=0.32, p=$ 0.81; Fig. 7D). Post hoc group comparisons by one-way ANOVA with Bonferroni's correction indicated that, like the CL paw, reaching performance of the AAVshPTEN/fibrin group with the IL paw was significantly better than the other groups at steps $1-5$

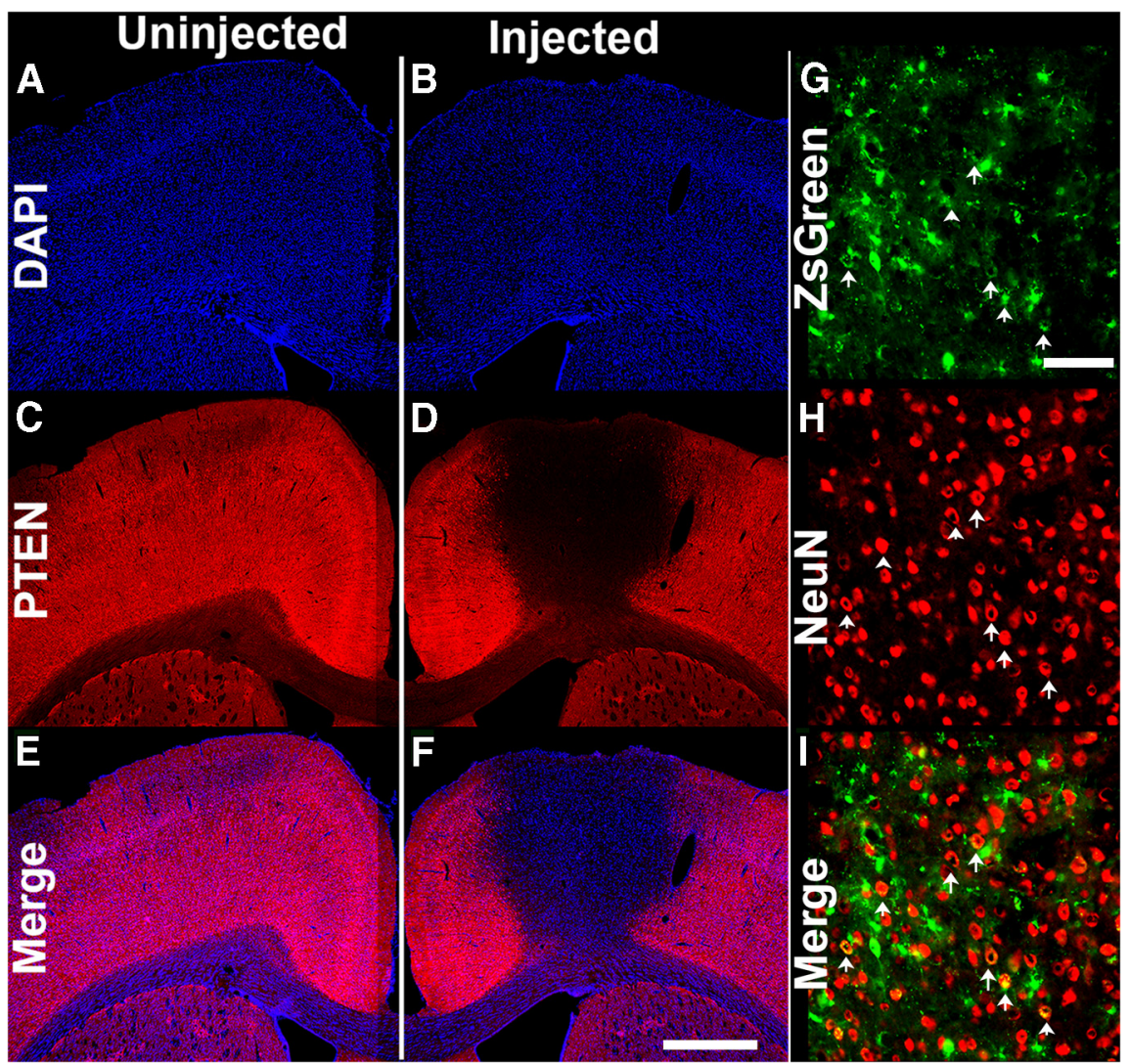

Figure 5. PTEN suppression is maintained for 15 weeks after multiple AAVshPTEN cortical injections. $A, B$, DAPI-stained coronal cortical section. $A, C, E$, Uninjected hemisphere. $\boldsymbol{B}, \boldsymbol{D}, \boldsymbol{F}$, Injected hemisphere from the same rat. $\boldsymbol{C}, \boldsymbol{D}$, PTEN immunofluorescence in the same sections as in $\boldsymbol{A}, \boldsymbol{B}$. $\boldsymbol{E}, \boldsymbol{F}$, Merged image of $\boldsymbol{A}, \boldsymbol{C}, \boldsymbol{B}$, and $\boldsymbol{D}$, respectively; $\boldsymbol{G}$, High-magnification view of $Z$ s Green fluorescence from the same animal in $\boldsymbol{A}$. $\boldsymbol{H}$, High-magnification view of NeuN immunofluorescence in the area of PTEN deletion (same section as in $\mathbf{G}$ ). $\boldsymbol{I}$, Merged image of $\boldsymbol{G}$ and $\boldsymbol{H}$; white arrows indicate cells that are positive for both ZsGreen and NeuN. Scale bars: $\boldsymbol{A}-\boldsymbol{F}, 1 \mathrm{~mm} ; \boldsymbol{G}-\boldsymbol{I}, 100 \mu \mathrm{m}$.

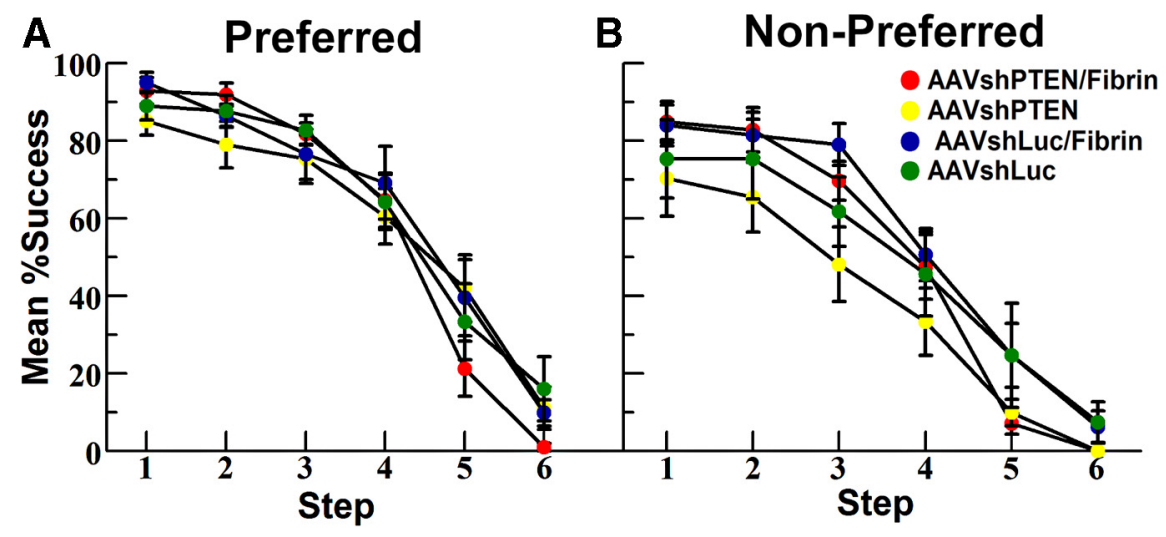

Figure 6. Preinjury performances of groups in the staircase-reaching task. Presurgery success rates at different steps for the "preferred" $(\boldsymbol{A})$ and "nonpreferred" (B) paw. Steps are numbered 1-6, with 1 being the highest step nearest the platform. Values are group means \pm SE. Green circles indicate AAVshLuc $(n=9)$; blue circles, AAVshLuc/fibrin $(n=9)$; yellow circles, $\operatorname{AAVshPTEN~}(n=9)$; red circles, $\operatorname{AAVshPTEN/fibrin}(n=11)$.

( $p<0.0001$ for all comparisons; Fig. $7 D$, asterisks). Interestingly, the AAVshLuc/fibrin group was also significantly better than the AAVshPTEN and AAVshLuc groups at steps 1-5 (Fig. 7D, daggers and carets; see legend for statistics for each time point). This is different from the CL paw, in which the performance of the AAVshLuc/fibrin group did not differ significantly from the AAVshPTEN and AAVshLuc groups. Similar to the CL paw, the performances of the AAVshPTEN and AAVshLuc groups with the IL paw did not differ significantly. 


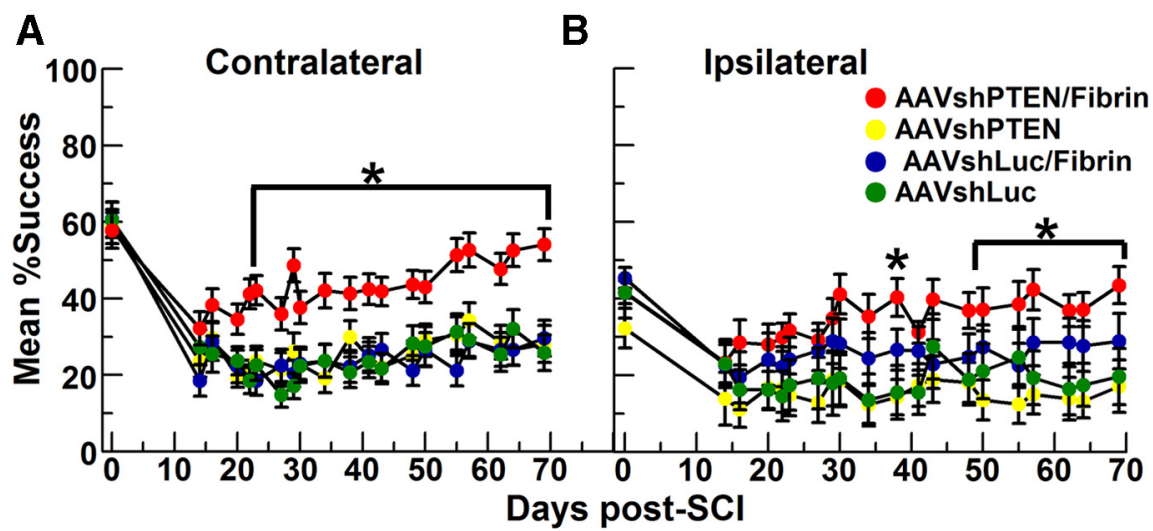

C

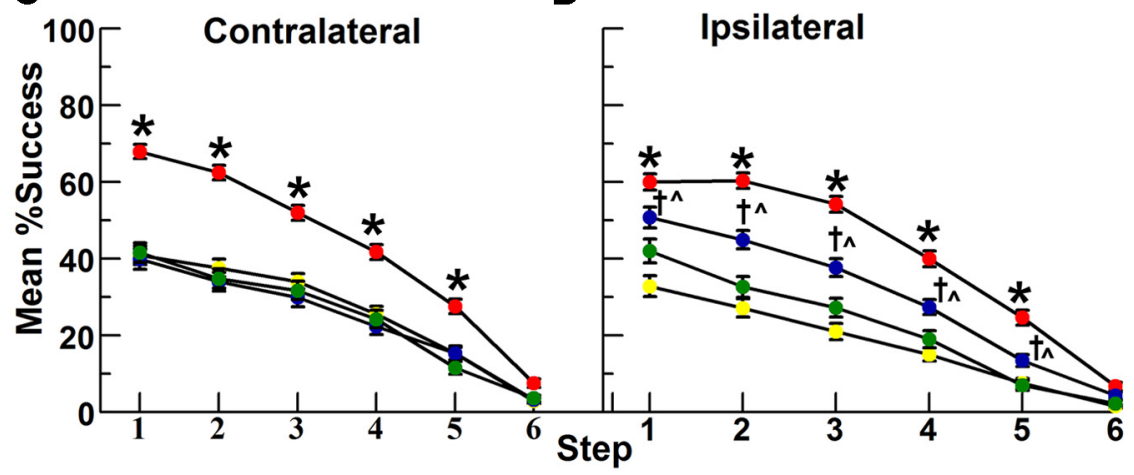

Figure 7. Rats that received AAVshPTEN and salmon fibrin exhibited greater recovery of forepaw function after cervical SCI. Format of graphs and group numbers are as in Figure 6. Values are group means $\pm \mathrm{SE}$. $A, B$, Graphs illustrating the mean percentage success over time for the $\mathrm{CL}$ paw versus the IL paw to the vector injections for different experimental groups. Asterisk in $A$ indicates significant differences between the AAVshPTEN/fibrin group versus other groups (one-way ANOVA with Bonferroni's correction, $p<0.0001$ ). Asterisks in B indicate significant differences between the AAVshPTEN/fibrin group versus other groups as follows: 38 and 55-57 dpi, $p=0.001 ; 50 \mathrm{dpi}, p=0.002 ; 62-64 \mathrm{dpi}, p=0.002 ; 69 \mathrm{dpi}, p=0.004 ;$ and $71 \mathrm{dpi}, p<0.0001$. $C$ $\boldsymbol{D}$, Mean percentage success for different steps (1-6) from all dpi for $C L(\boldsymbol{C})$ and IL $(\boldsymbol{D})$ paws. Asterisks in $\boldsymbol{C}$ and $\boldsymbol{D}$ indicate significant differences between the AAVshPTEN/fibrin group versus other groups $(p<0.0001)$. $\boldsymbol{D}$, Daggers indicate significant differences between AAVshLuc/fibrin and AAVshPTEN groups on steps $1-4(p<0.0001)$ and step 5, $(p=0.003)$; carets indicate significant differences between the AAVshLuc/fibrin and AAVshLuc groups on step $1(p=0.004)$, steps $2-3(p<0.0001)$, step 4 ( $p=$ $0.001)$, and step $5(p=0.007)$.

Rats receiving AAVshPTEN and salmon fibrin had larger numbers of BDA-labeled axons at the edge of the SCI lesion We counted the number of BDA-labeled axons $\left(\mathrm{BDA}^{+}\right)$in serial sagittal sections of the spinal cord at intervals of $0.5 \mathrm{~mm}$ from $5 \mathrm{~mm}$ rostral $(-5.0$ $\mathrm{mm})$ to the lesion edge $(0 \mathrm{~mm})$ and to $5 \mathrm{~mm}$ caudal $(5.0 \mathrm{~mm})$. The number of $\mathrm{BDA}^{+}$axons at each distance was expressed as a proportion of the total number of $\mathrm{BDA}^{+}$axons counted in cross-sections rostral to the lesion and is referred to as the $\mathrm{BDA}^{+}$axon index. The total number of $\mathrm{BDA}^{+}$axons in the rostral sections was not significantly different between groups (Fig. 8E).

Consistent with the fact that CST axons extend longitudinally along the dorsal column, the $\mathrm{BDA}^{+}$axon index remained almost constant in all groups from $-5 \mathrm{~mm}$ to $\sim-2 \mathrm{~mm}$ from the lesion (Fig. $8 A-C, F$ ). The number of $\mathrm{BDA}^{+}$axons in the AAVshLuc and AAVshLuc/fibrin groups decreased sharply approaching the lesion, as expected due to dieback of transected axons. Very few BDA-labeled axons were observed in the counting site just rostral to the lesion (Fig. $8 C, F$ ).

In contrast, in the AAVshPTEN- and AAVshPTEN/fibrin-treated groups, the number of $\mathrm{BDA}^{+}$axons remained constant from $-5 \mathrm{~mm}$ to $-1 \mathrm{~mm}$ and $\sim-0.5 \mathrm{~mm}$, respectively (Fig. $8 B, C, F$ ). One-way ANOVA revealed statistically significant group differences in $\mathrm{BDA}^{+}$axon index in counting sites $-1.0 \mathrm{~mm}(F=3.5, p=0.045),-0.5 \mathrm{~mm}(F=3.5, p=$ $0.042)$, and $0 \mathrm{~mm}(F=7.9, p=0.003)$ (Fig. $8 F)$. Post hoc analysis with Bonferroni's multiple-comparison test revealed that the axon index for the AAVshPTEN group was not statistically different at $-1.0 \mathrm{~mm},-0.5$, and $0 \mathrm{~mm}$ from that of the AAVshLuc group $(p=0.228, p=0.228$, and $p=0.057$, respectively) or from that of the AAVshLuc/fibrin group $(p=0.102, p=0.104$, and $p=0.0 .045$, respectively). The axon index for the AAVshPTEN/Fibrin group was statistically different at $-1.0 \mathrm{~mm},-0.5$, and $0 \mathrm{~mm}$ from that of the AAVshLuc group ( $p=0.005, p=0.004$, and $p=0.003$, respectively) and from that of the AAVshLuc/Fibrin group ( $p=0.007, p=$ 0.006 , and $p=0.010$, respectively). At these same counting sites, the axon index of the AAVshPTEN/Fibrin group was not statistically different from that of the AAVshPTEN group $(p=0.314, p=0.121$, and $p=0.111$, respectively). Moreover, in most of the AAVshPTENtreated rats, there was an obvious bloom of $\mathrm{BDA}^{+}$axons near the lesion edge (Fig. $8 \mathrm{~B}, \mathrm{C}$ box, $D)$. The pattern seen here in rats treated with AAVshPTEN was similar, although less pronounced, than that seen in previous studies of regenerative growth of CST axons in mice after conditional genetic deletion of PTEN (Liu et al., 2010).

Only a few $\mathrm{BDA}^{+}$axons circumvented the lesion, and these were seen only in the AAVshPTEN/fibrin group. A few BDA-labeled axons crossed through the lesion in the AAVshPTEN/fibrin group (Fig. $8 C, D$, asterisk). Only a few $\mathrm{BDA}^{+}$axons were observed caudal to the lesion in any of the groups and these were for the most part in the ventral column in the location of the ventral CST (Fig. $8 C, D$, double asterisk).

\section{Lesion volume}

One possible explanation for the higher performance of the AAVshPTEN/fibrin group is that the lesion was smaller in this group for some reason. To assess this, lesion volumes were assessed in the same animals as used for BDA analysis. In all groups, the lesion site was characterized by a cystic cavity surrounded by areas of scar tissue. In addition, a fibrous scar formed above the injury where the dura mater was cut and was visible dorsal to the cavity in all spinal cords examined (Fig. 9, white outlines). One-way ANOVA revealed no statistically significant differences in lesion volumes between groups $(\mathrm{df}=3 ; F=0.2168 ; p=0.8835)$. Most importantly, lesion volume was not smaller in the AAVshPTEN/ fibrin group, so differences in lesion size are unlikely to be the cause of the consistently better forelimb function in this group. One qualitative difference is that small tissue bridges were often present in the lesions of the fibrin-treated rats (Fig. 9A, asterisk).

\section{Discussion}

Our goals in this study were to assess whether shRNA-mediated PTEN suppression in mature cortical motoneurons can enable CST axon regeneration after cervical SCI, whether PTEN suppression enhanced recovery of voluntary motor function, and whether the combination of PTEN suppression and manipulations at the injury site (injection of salmon fibrin) would have a combinatorial effect. Our results indicate that AAV-shRNAmediated suppression of PTEN in adult cortical motoneurons does enhance regenerative growth of CST axons after SCI. Although PTEN suppression alone did not improve recovery of voluntary 
forelimb motor function, the combination of AAVshRNA-mediated suppression of PTEN with salmon fibrin injection into the injury site was associated with improved forepaw-grasping function. To our knowledge, this is the first evidence that shRNAmediated PTEN suppression in the adult brain, when combined with salmon fibrin, can enhance functional recovery after SCI.

\section{AAVshPTEN is efficacious for PTEN knockdown in transduced cells of the motor cortex}

Injections of AAVshPTEN into the adult sensorimotor cortex effectively suppressed PTEN expression in the area of the injection, accompanied by increased pS6 phosphorylation in neurons lacking PTEN. PTEN depletion accompanied by pS6 activation was seen in a previous study in which AAV2-shPTEN was injected in developing mice (Zukor et al., 2013). However, the pattern of PTEN suppression in the motor cortex was different in the present study. Here, PTEN was suppressed throughout a defined zone, whereas in the previous study, the area of PTEN suppression contained intermixed PTEN-negative and PTENpositive cells. The most likely explanation for the difference in pattern is developmental age at the time of AAV injection because the pattern of suppression seen in Zukor et al. (2013) is very similar to what is seen with AAV-Cre injections into neonatal PTEN ${ }^{\mathrm{ff}}$ mice (unpublished observations).

\section{PTEN suppression enhances}

regenerative growth of CST axons

Our data are consistent with the notion that PTEN suppression in adult cortical motoneurons enhances regenerative growth of CST axons. This effect is further enhanced by treatment of the lesion site with salmon fibrin. It is important to be clear about the nature of the growth seen here. The lesion is a cervical dorsal hemisection, which interrupts CST axons in the dorsal and dorsolateral CST. In control rats, CST axons die back from the site of the injury, retreating for several hundred micrometers. In the control animals (AAVshLuc), dieback is seen as a decrease in the number of labeled CST axons approaching the lesion. In rats treated with AAVshPTEN and salmon fibrin, axon numbers are significantly higher in the areas just rostral to the lesion, some axons extend ventrally around the lesion, and a few extend through the lesion. This pattern is consistent with regenerative growth. The increased number of axons seen in the segments just rostral to the injury could also be due to prevention of axon dieback, but extension past the lesion can only be explained by regenerative growth.

In interpreting these data, it should be noted that there were more tract tracing failures than we typically obtain (rats with no or minimal CST labeling), reducing the number available for anatomical analysis. In addition, labeled axons were not as strongly labeled as is typical in our studies, probably because BDA

\section{B AAVshPTEN}
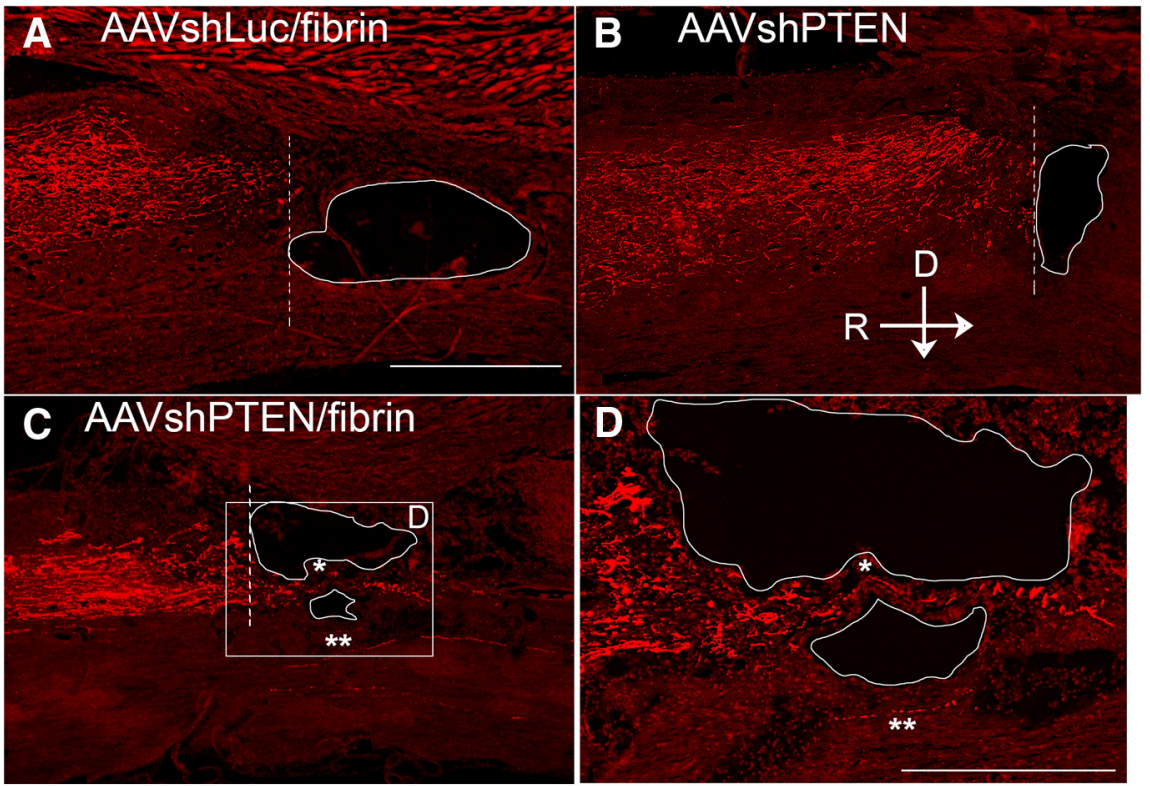

F

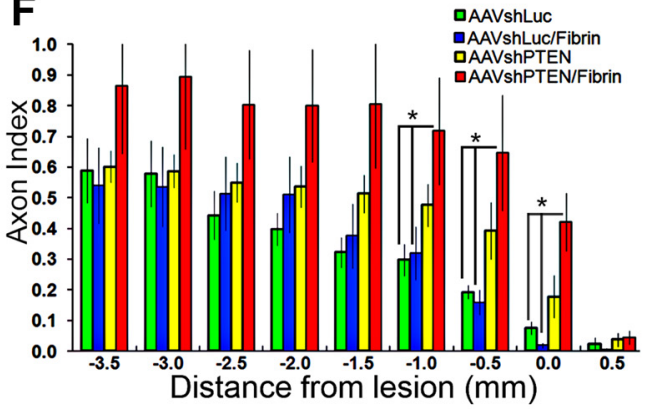

口AAVShLuc

GAVShLuC/Fib

$p=0.32$

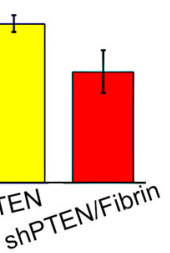

Distance from lesion $(\mathrm{mm})$

Figure 8. Larger numbers of BDA-labeled axons at the edge of the SCI lesion in rats treated with AAVshPTEN and salmon fibrin A-C, Sagittal sections illustrating BDA-labeled axons near the lesion sites in the different groups. Lesion cavity is outlined in white the rostral lesion edge expressed as an index of the number of BDA axons/total number of BDA axons in rostral cross-sections. Group sizes were as follows: AAVshLuc, $n=4$ (green bars); AAVshLuc/Fibrin, $n=4$ (blue bars); AAVshPTEN, $n=5$ (yellow bars); and AAVshPTEN/fibrin, $n=5$ (red bars). Asterisks indicate significant differences between groups. Scale bars: $\boldsymbol{A}-\boldsymbol{C}, 1 \mathrm{~mm} ; \boldsymbol{D}, 0.5 \mathrm{~mm}$.

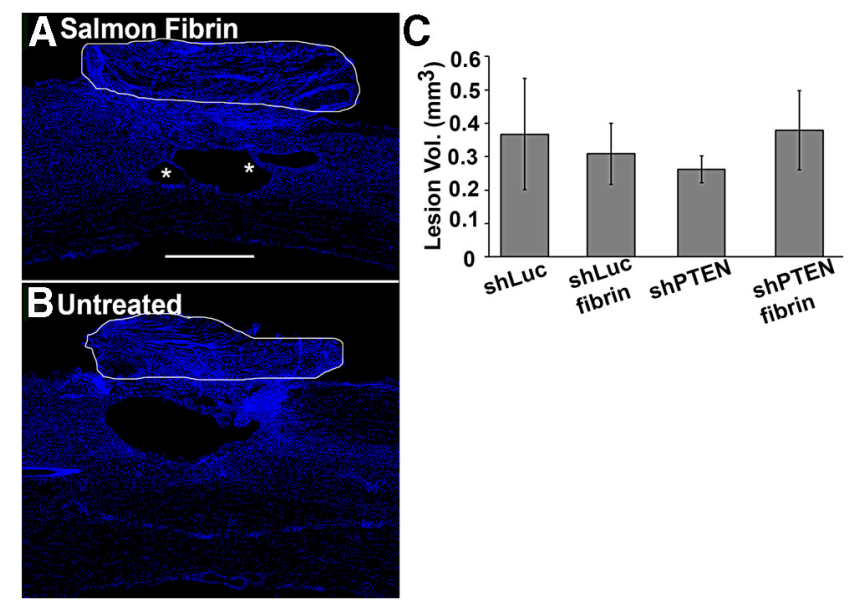

Figure 9. No significant differences in lesion volumes were seen between groups. $\boldsymbol{A}, \boldsymbol{B}$, Representative DAPI-stained sagittal sections through the lesion area from a rat in the AAVshPTEN/fibrin versus the AAVshPTEN groups, respectively. Fibrous scar dorsal to the cavity is outlined in white. Asterisks in $\boldsymbol{A}$ indicate thin cellular bridges. C, Quantification of lesions volumes in the four groups (means $\pm \mathrm{SE}$ ). 
staining was done using slide-mounted rather than free-floating sections. This was done to preserve the architecture at the injury site and to retain any material in the injury cavity that might be due to the fibrin treatment. Despite these caveats, differences in CST axon numbers were statistically significant between groups.

The axon extension seen here is similar, but appears less extensive, to what is seen with neonatal deletion of PTEN, where axons extend for several millimeters beyond a lesion, even growing through the injury site of a complete crush injury (Liu et al., 2010; Zukor et al., 2013). The difference could be due to either the animal age at the time of AAVshPTEN injections or the rodent species. Neuronal migration and integration of neurons into circuits is incomplete at P0 (Sun et al., 2002) and are dependent, in part, on the phosphoinositide 3-kinase (PI3K) signaling pathway (Polleux et al., 2002). PTEN, which inactivates PI3K through dephosphorylation (Maehama and Dixon, 1998), plays a role in neural circuit development and maintenance (van Diepen and Eickholt, 2008). Moreover, PTEN expression in the mouse brain starts around $\mathrm{P} 0$ and is expressed at a lower level than in the adult brain (Lachyankar et al., 2000). Therefore, suppression of PTEN at P0 may restrict neurons to a more immature/grow permissive state. Therefore, the maximal extent of regeneration enabled by PTEN gene silencing in adult animals may be less than that after neonatal PTEN deletion and may require additional gene manipulations. Indeed, in studies using the optic nerve model, co-deletion of PTEN and SOCS3 activates different cellular pathways and stimulates more robust long-distance axon extension than deletion of either gene alone (Sun et al., 2011). It remains to be seen whether the same applies to cortical motoneurons.

It is also possible that differences in regenerative growth seen here compared with Zukor et al. (2013) are because we used rats, whereas they used mice. This is significant because, in the rat, a cavity forms in the lesion core that presents a physical barrier, whereas in mice, the lesion site is filled in with a connective tissue matrix (Guth et al., 1999). The expectation that cavitation might limit axon growth led to the approach of combining AAVshPTEN with salmon fibrin injection into the injury. Consistent with this, the highest numbers of axons were observed in rats that received both of AAVshPTEN and salmon fibrin. Previous studies have shown that salmon fibrin promotes neuroprotection; sealing of the blood-brain barrier, thereby decreasing immune cell influx; and greater neurite extension than other biomaterials, including human fibrin and Matrigel (Ju et al., 2007; Uibo et al., 2009; King et al., 2010). Our results are consistent with the notion that salmon fibrin favorably changes the lesion environment such that axon dieback and axon extension may be decreased and increased, respectively. Because salmon fibrin is degraded within 2 weeks of injection, it is unlikely that it was incorporated into the observed tissue bridges (Sharp et al., 2012). Although narrow tissue bridges were observed, the salmon fibrin did not appear to stimulate cellular repair of the lesion, because the lesion sizes were similar to control rats. These results are consistent with a previous study (Sharp et al., 2012), but should be taken with caution because the comparison was not sufficiently powered because of the small number of subjects.

\section{Enhanced recovery of voluntary forepaw function after cervical SCI in rats that received AAVshPTEN and salmon fibrin}

The fact that rats that received AAVshPTEN and salmon fibrin exhibited regenerative growth CST axons and enhanced functional recovery invites the speculation that the two are related. Nevertheless, our results do not definitively establish this cause- effect relationship. The enhanced recovery begins quite early post-lesion (by 3 weeks after SCI), which is unlikely to be related to long-distance axon regeneration, but could reflect local, shortdistance growth. The continued functional improvement between 3 and 10 weeks is consistent with continued growth, but could be due to other causes, including learning to use modified connections.

Our anatomical results suggest that PTEN suppression can enhance regenerative growth of axons, but rats treated with AAVshPTEN alone did not exhibit enhanced motor function. This is consistent with the consensus that manipulations that enhance axon growth will have to be combined with manipulations to overcome the inhibitory environment of the lesion. Our data suggest that salmon fibrin may make the lesion area more permissive to axon growth, but does not lead long-distance growth of axons past the lesion. Cellular transplants may be more effective for bridging and overcoming the inhibitory environment of the lesion site (Lu et al., 2012).

The notion of targeting multiple aspects of SCI is not new, and combination therapies for treatment of SCI, including strategies that include biomaterial scaffolding, have been described previously (McCreedy and Sakiyama-Elbert, 2012; Krishna et al., 2013; Zhao and Fawcett, 2013). However, to date, few approaches have targeted diminished regenerative capacity at the level of the neuronal cell body in the brain. One such study reported significant axon regeneration and forelimb function recovery in rats that received cortical injections of the protein kinase $\mathrm{C}$ inhibitor GÖ6976 combined with lesion site injections of a lentivirus expressing ChABC (Wang et al., 2013). Our results suggest that combining manipulations at the level of the cell body with interventions at the lesion site are more efficacious than either manipulation alone.

In conclusion, our data demonstrate that an shRNA targeting PTEN can suppress PTEN expression in the adult rat cortex for weeks and enhance regenerative growth of CST axons in the injured spinal cord. Moreover, PTEN suppression can contribute to enhanced recovery of voluntary motor function after a cervical SCI when combined with salmon fibrin injection in the lesion. It remains to be seen whether interventions in more clinically relevant time frames (days or weeks after injury) may also be efficacious.

\section{References}

Afshari FT, Kappagantula S, Fawcett JW (2009) Extrinsic and intrinsic factors controlling axonal regeneration after spinal cord injury. Expert Rev Mol Med 11:e37. CrossRef Medline

Aschauer DF, Kreuz S, Rumpel S (2013) Analysis of transduction efficiency, tropism and axonal transport of AAV serotypes 1, 2, 5, 6, 8 and 9 in the mouse brain. PLoS One 8:e76310. CrossRef Medline

Cearley CN, Wolfe JH (2006) Transduction characteristics of adenoassociated virus vectors expressing cap serotypes 7, 8, 9, and Rh10 in the mouse brain. Mol Ther 13:528-537. CrossRef Medline

Cregg JM, DePaul MA, Filous AR, Lang BT, Tran A, Silver J (2014) Functional regeneration beyond the glial scar. Exp Neurol 253:197-207. CrossRef Medline

Ferguson TA, Son YJ (2011) Extrinsic and intrinsic determinants of nerve regeneration. J Tissue Eng 2. CrossRef Medline

Fonoff ET, Pereira JF Jr, Camargo LV, Dale CS, Pagano RL, Ballester G, Teixeira MJ (2009) Functional mapping of the motor cortex of the rat using transdural electrical stimulation. Behav Brain Res 202:138-141. CrossRef Medline

Guth L, Zhang Z, Steward O (1999) The unique histopathological responses of the injured spinal cord: implications for neuroprotective therapy. Ann N Y Acad Sci 890:366-384. CrossRef Medline

Ju YE, Janmey PA, McCormick ME, Sawyer ES, Flanagan LA (2007) Enhanced neurite growth from mammalian neurons in three-dimensional salmon fibrin gels. Biomaterials 28:2097-2108. CrossRef Medline 
King VR, Alovskaya A, Wei DY, Brown RA, Priestley JV (2010) The use of injectable forms of fibrin and fibronectin to support axonal ingrowth after spinal cord injury. Biomaterials 31:4447-4456. CrossRef Medline

Klein A, Dunnett SB (2012) Analysis of skilled forelimb movement in rats: the single pellet reaching test and staircase test. Curr Protoc Neurosci Chapter 8:Unit8.28. CrossRef Medline

Kloth V, Klein A, Loettrich D, Nikkhah G (2006) Colour-coded pellets increase the sensitivity of the staircase test to differentiate skilled forelimb performances of control and 6-hydroxydopamine lesioned rats. Brain Res Bull 70:68-80. CrossRef Medline

Krishna V, Konakondla S, Nicholas J, Varma A, Kindy M, Wen X (2013) Biomaterial-based interventions for neuronal regeneration and functional recovery in rodent model of spinal cord injury: a systematic review. J Spinal Cord Med 36:174-190. CrossRef Medline

Lachyankar MB, Sultana N, Schonhoff CM, Mitra P, Poluha W, Lambert S, Quesenberry PJ, Litofsky NS, Recht LD, Nabi R, Miller SJ, Ohta S, Neel $\mathrm{BG}$, Ross AH (2000) A role for nuclear PTEN in neuronal differentiation. J Neurosci 20:1404-1413. Medline

Liu K, Lu Y, Lee JK, Samara R, Willenberg R, Sears-Kraxberger I, Tedeschi A, Park KK, Jin D, Cai B, Xu B, Connolly L, Steward O, Zheng B, He Z (2010) PTEN deletion enhances the regenerative ability of adult corticospinal neurons. Nat Neurosci 13:1075-1081. CrossRef Medline

Lu P, Blesch A, Graham L, Wang Y, Samara R, Banos K, Haringer V, Havton L, Weishaupt N, Bennett D, Fouad K, Tuszynski MH (2012) Motor axonal regeneration after partial and complete spinal cord transection. J Neurosci 32:8208-8218. CrossRef Medline

Maehama T, Dixon JE (1998) The tumor suppressor, PTEN/MMAC1, dephosphorylates the lipid second messenger, phosphatidylinositol 3,4,5trisphosphate. J Biol Chem 273:13375-13378. CrossRef Medline

McCreedy DA, Sakiyama-Elbert SE (2012) Combination therapies in the CNS: Engineering the environment. Neurosci Lett 519:115-121. CrossRef Medline

Montoya CP, Campbell-Hope LJ, Pemberton KD, Dunnett SB (1991) The "staircase test" a measure of independent forelimb reaching and grasping abilities in rats. J Neurosci Methods 36:219-228. CrossRef Medline

Neafsey EJ, Bold EL, Haas G, Hurley-Gius KM, Quirk G, Sievert CF, Terreberry RR (1986) The organization of the rat motor cortex: a microstimulation mapping study. Brain Res 396:77-96. CrossRef Medline

Nielson JL, Strong MK, Steward O (2011) A reassessment of whether corti- cal motor neurons die following spinal cord injury. J Comp Neurol 519: 2852-2869. CrossRef Medline

Park KK, Liu K, Hu Y, Kanter JL, He Z (2010) PTEN/mTOR and axon regeneration. Exp Neurol 223:45-50. CrossRef Medline

Polleux F, Whitford KL, Dijkhuizen PA, Vitalis T, Ghosh A (2002) Control of cortical interneuron migration by neurotrophins and PI3-kinase signaling. Development 129:3147-3160. Medline

Sharp KG, Dickson AR, Marchenko SA, Yee KM, Emery PN, Laidmåe I, Uibo R, Sawyer ES, Steward O, Flanagan LA (2012) Salmon fibrin treatment of spinal cord injury promotes functional recovery and density of serotonergic innervation. Exp Neurol 235:345-356. CrossRef Medline

Strong MK, Blanco JE, Anderson KD, Lewandoski G, Steward O (2009) An investigation of the cortical control of forepaw gripping after cervical hemisection injuries in rats. Exp Neurol 217:96-107. CrossRef Medline

Sun F, Park KK, Belin S, Wang D, Lu T, Chen G, Zhang K, Yeung C, Feng G, Yankner BA, He Z (2011) Sustained axon regeneration induced by codeletion of PTEN and SOCS3. Nature 480:372-375. CrossRef Medline

Sun XZ, Takahashi S, Cui C, Zhang R, Sakata-Haga H, Sawada K, Fukui Y (2002) Normal and abnormal neuronal migration in the developing cerebral cortex. J Med Invest 49:97-110. Medline

Uibo R, Laidmäe I, Sawyer ES, Flanagan LA, Georges PC, Winer JP, Janmey PA (2009) Soft materials to treat central nervous system injuries: Evaluation of the suitability of non-mammalian fibrin gels. Biochim Biophys Acta 1793:924-930. CrossRef Medline

van Diepen MT, Eickholt BJ (2008) Function of PTEN during the formation and maintenance of neuronal circuits in the brain. Dev Neurosci 30:59-64. CrossRef Medline

Wang X, Hu J, She Y, Smith GM, Xu X-M (2013) Cortical PKC inhibition promotes axonal regeneration of the corticospinal tract and forelimb functional recovery after cervical dorsal spinal hemisection in adult rats. Cereb Cortex. Advance online publication. doi:10.1093/cercor/bht162. CrossRef Medline

Zhao RR, Fawcett JW (2013) Combination treatment with chondroitinase $\mathrm{ABC}$ in spinal cord injury-breaking the barrier. Neurosci Bull 29:477-483. CrossRef Medline

Zukor K, Belin S, Wang C, Keelan N, Wang X, He Z (2013) Short hairpin RNA against PTEN enhances regenerative growth of corticospinal tract axons after spinal cord injury. J Neurosci 33:15350-15361. CrossRef Medline 